\title{
LATEST DEVELOPMENT IN ADVANCED SENSORS AT KENNEDY SPACE CENTER (KSC)
}

\section{Jose M. Perotti}

Acting Lead, Transducers and Data Acquisition Group, National Aeronautics and Space Administration Kennedy Space Center, Florida 32899, USA

jose.perotti-1@ksc.nasa.gov

\begin{abstract}
Inexpensive space transportation system must be developed in order to make space flight more affordable. To achieve this goal, there is a need to develop inexpensive smart sensors to allow autonomous checking of the health of the vehicle and associated ground support equipment, warn technicians or operators of an impending problem and facilitate rapid vehicle pre-launch operations. The Transducers and Data Acquisition group at Kennedy Space Center has initiated an effort to study, research, develop and prototype inexpensive smart sensors to accomplish these goals. Several technological challenges are being investigated and integrated in this project multi-discipline sensors; self-calibration, health selfdiagnosis capabilities embedded in sensors; advanced data acquisition systems with failure prediction algorithms and failure correction (self-healing) capabilities.
\end{abstract}

\section{INTRODUCTION}

An inexpensive space transportation system must be developed in order to make space flight more affordable. To achieve this goal, systems designed for space transportation vehicles must include unique features not available in

\author{
Anthony J. Eckhoff \\ Manager, Transducers and Hazardous Gas Detection \\ Dynacs Incorporated \\ Kennedy Space Center, Florida 32899, USA \\ anthony.eckhoff-1@ksc.nasa.gov
}

the present vehicles. There is a need to develop inexpensive smart sensors to allow autonomous checking the health of the vehicle and associated ground support equipment, warn technicians or operators of an impending problem and facilitate rapid vehicle pre-launch operations.

Sensors and sensing systems will have to be capable of monitoring their own health, have long calibration cycles, high reliability, and be multi-parameter in nature. They will also need to be robust to survive space environment, low in weight, volume and have reduced power consumption. Furthermore, they will have to be inexpensively mass-produced.

Currently there are very few sensors available that meet the above requirements to support integrated space launch vehicle sensing systems and automated ground support systems. Many of the parameters needing to be sensed in a launch system are common from vehicle to vehicle (i.e. temperature, pressure, acceleration) while other parameters are only similar in principle, such as chemical species sensing. As an industry, we have to start delineating sensing technologies needing development, as well as to identify technology gaps needed for future developments. Furthermore, a common standard is required for sensor

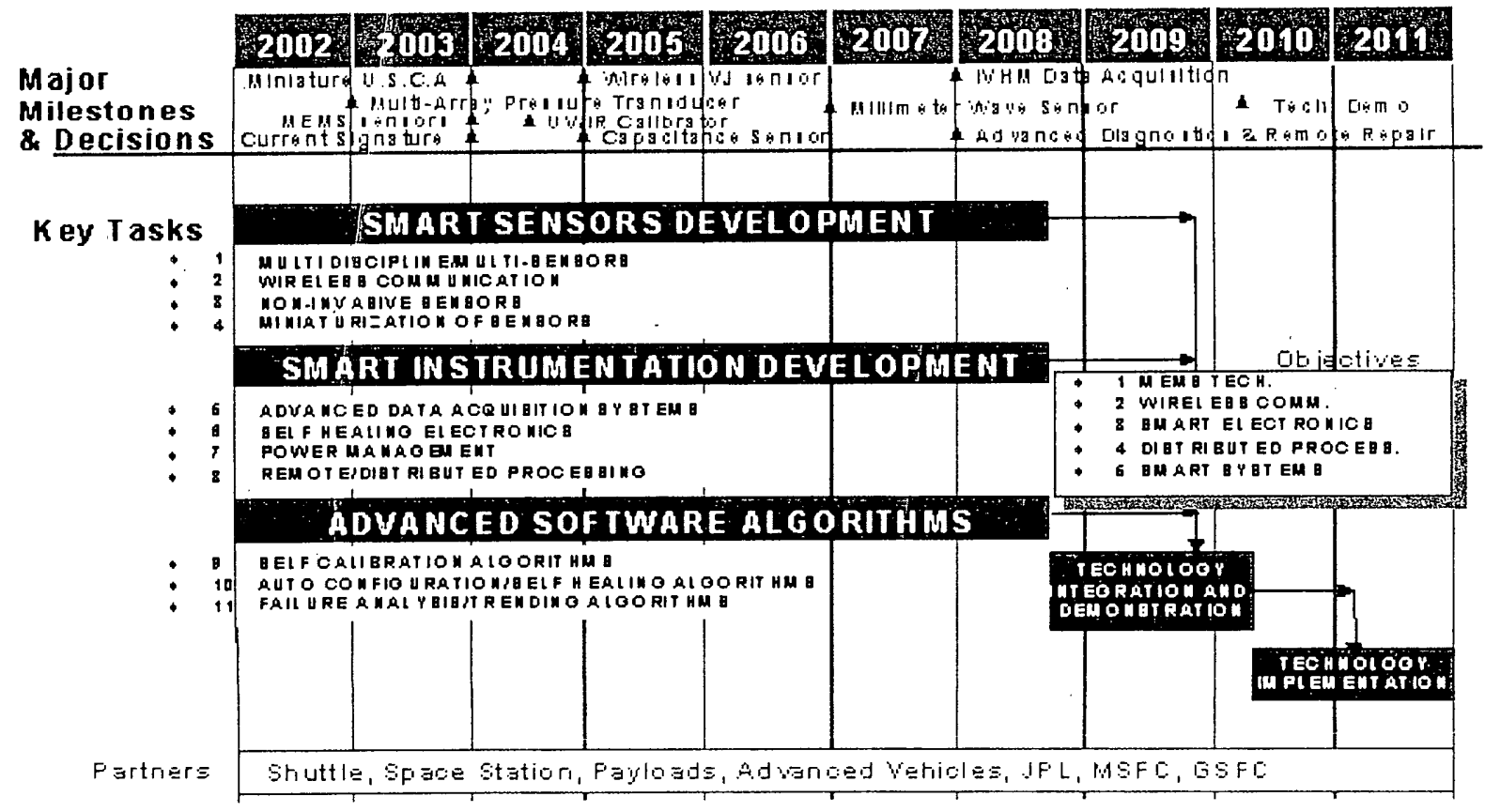

Figure 1. - Advanced Sensors and Electronics proposed Road- 
analog signal and digital system communication, and command and control, thus further reducing the cost of implementation.

The Transducers and Data Acquisition group at Kennedy Space Center studied these goals and requirements about three years ago and formulated a group's roadmap to align to these needs (Figure 1). The group has initiated an effort to study, research, develop and prototype inexpensive smart sensors to accomplish the goals mentioned above. Several technological challenges are being investigated and integrated in this project. Among others, they are:

- The development of multi-sensor array systems

- The development of multi-discipline sensors

- The development of self-calibration, health selfdiagnosis capabilities embedded in sensors.

- The development of advanced data acquisition systems with failure prediction algorithms and failure correction (self-healing) capabilities.

This article will address only a few of the efforts pursued by this group in the area of transducers and sensors development. A parallel effort is being pursued in the area of data acquisition systems.

The authors will also like to recognize that several other efforts in the area of sensors' development are currently going on at Kennedy Space Center that are not part of this group and are not included in this article.

\section{MULTI-DISCIPLINE, MULTI-SENSOR ARRAY TRANSDUCERS DEVELOPMENT EFFORTS}

Two main development efforts are being presently pursued at our group related to this discipline: (a) the development of a multi-sensor array pressure transducer and (b) the development of an extreme wind sensor for hurricane force winds measurements.

The development of the Multi-Sensor Array (MSA) pressure transducer is headed by the above-mentioned authors in conjunction with Dr. Christopher Immer and Dr. John Lane of DYNACS Incorporated.

The goal of this project is to demonstrate a measurable increase in transducer's reliability as well as to expand their calibration cycles by combining the use of nultisensor arrays with in-house smart software algorithms. We want to quantify the relationship between numb er of sensors and the associated improvement in sensor life, calibration, and reliability.

The ability to have multiple sensors can be impleme: ited in numerous ways, the most direct of which is to sim sly increase the number of sensors of a particular type at he measurement site. Another method, which takes adva ntage of the reduced size of micro-electronic sensors, is o pack several discrete sensors onto a single circuit boar:!
Going one level smaller, multiple sensor dies can be combined into a single IC package (Multi-Chip-Modules MCM). Finally, multiple sensors can be fabricated directly onto a single die, using advanced technologies such as micro-electro-mechanical systems (MEMS). Whatever the technique used to implement a multi-sensor array (MSA), the question to answer is: "is it better, and if so, how much better?"

To initially address this question, a computer-generated experiment (Monte Carlo simulation) was first performed.

An arricy (or cluster) of $N$ sensors were assigned random failures, such that the statistical mean of the failure mode remained constant (zero mean drift). A life extension factor (LEF; was defined as a percentage of the life of a single sensor. When The LEF was plotted as a function of $N$ (the number of sensors of the array), a maximum LEF of 3 was reache 1 beyond $N>30$, as shown in Figure 2 .

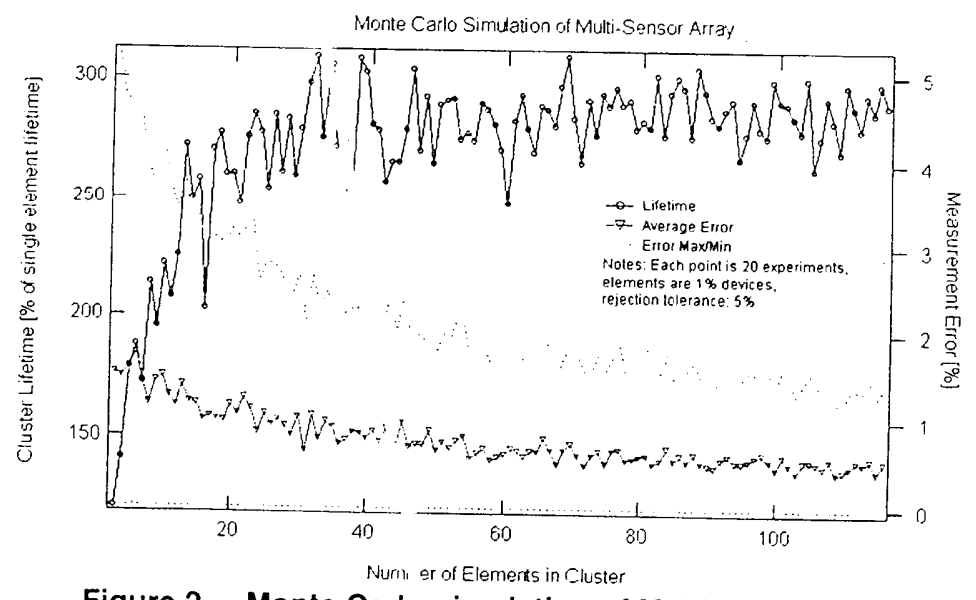

Figure 2. - Monte Carlı simulation of Multi-Sensor , Irray

The next step, following tile computer simulation, was to perform a test using real de vices. For this experiment, we selected an array of discret $\epsilon$ pressure sensors attached to a single printed circuit board (pcb). The pcb was approximately one-inch square, con aining eight, 8-lead IC pressure transducers. To accels rate sensor failures, the experiment was conducted usin s an environmental chamber. The initial test temperature wis set to $125 \mathrm{C}$.

Every 24 hours, all sensors wire returned to ambient temperature of $27 \mathrm{C}$, and the o itput measured at ambient pressure. Once per week, a prissure calibration was made of all 8 sensors at 5 pressures: 3, 6, 9, 12, and 15 PSIA.

The test was scheduled to be stopped when all but two sensors had "failed", or until the elapsed test time have exceeded 1000 hrs. The test was stopped with no hard failures detected on the pressure sensors. A divergence from nominal performance parameters was detected on these transducers to support utilizing Accelerated Stress Testing (AST) for this experiment. A second set of temperature tests was performed at an environment tempera- 
ture of $80 \mathrm{C}$. No divergence from nominal performance parameters was detected on these transducers.

When more than one test temperature is used (more than one test group), an Arrhenius model analysis can be performed. Using data from each several groups, an "activation energy" can be extracted using the Arrhenius Equation, as shown in Equation 1. Using standard statistical methods, a calibration error distribution can be estimated which is a function of time and temperature. The drift of the mean and variance of this distribution can be determined (drift of mean corresponds to systematic failure modes, increase in variance corresponds to random failure modes).

Using the initial pressure calibration performed, the MSA was processed with an in-house developed multi-sensor array algorithm (MSAA), and compared to the measurement error of each individual sensor. This software resembles the statistical process used in the Monte Carlo simulation. A polling logic, combined with a sensor weighting algorithm and a sensor exclusion algorithm (to eliminate outliers from the decision-making process) is embedded in the sensor to provide a calculated output.

Additional development and testing is required in this project. Preliminary efforts to produce field-rated prototypes are under way. Prototypes will be installed in the

Equation 1. - Model for Estimating Absolute Device Lifetimo

$$
\lambda=\lambda_{0} e^{-Q / T+\sum_{i} \alpha_{i} S_{i}}
$$

\section{$\lambda$ Degradation Rate}

$Q$ Activation Energy of Failure Mechanism

$T$ Absolute Temperature

$S_{i} i^{\text {th }}$ Environmental Stress Factor

$\lambda_{0}, \alpha_{i}, Q$ Model Parameters (curve fitting parameters)

field (KSC launch Pads) and monitored over time to verify simulation results.

The next development effort in this discipline area is the Extreme Velocity Wind Measuring System (EVWMS). This effort is headed by the above-mentioned authors in conjunction with Mr. Jan Zysko of NASA- KSC, Mr. Norman Blalock and Mr. John Randazzo of DYNACS Incorporated.

The current wind sensors used at remote KSC locations for wind speed detection lack in two main areas. First, rotating cup or vane type anemometers have a high maintainability due to the wear-and-tear of their moving components. Secondly, there is a high degree of failure associated with such systems due to damage from extreme wind conditions. A key reference is Hurricane Andrew in 1993, when no ground-based wind sensors survived the path of the storm. Hence, there are no verifiable measurements of the highest winds generated by Andrew.

The EVWMS, referred to as the 3-D venturi wind sensor, was developed to provide measurements of extreme winds at various locations around KSC. The 3-D Venturi wind sensor measures wind speed through the use of pressure measurements across a shaped surface. The basic form is that of a typical streamlined venturi profile (a double-inflection curve) revolved 360 degrees about an axis passing vertically through the center of the profile. The profile has a series of instrumented ports located near the center and periphery to allow for pressure measurements along the surface (Figure 3 ). The wind speed is calculated from applying Bernoulli's law to the pressure change created between the ports. $\left(P=1 / 2 \rho^{*} k^{*}\right.$ $\mathrm{V}^{2}$ ). Wind direction is derived from the pressure profile distributed over the surface. Additionally, temperature and relative humidity measurements are incorporated into the design.

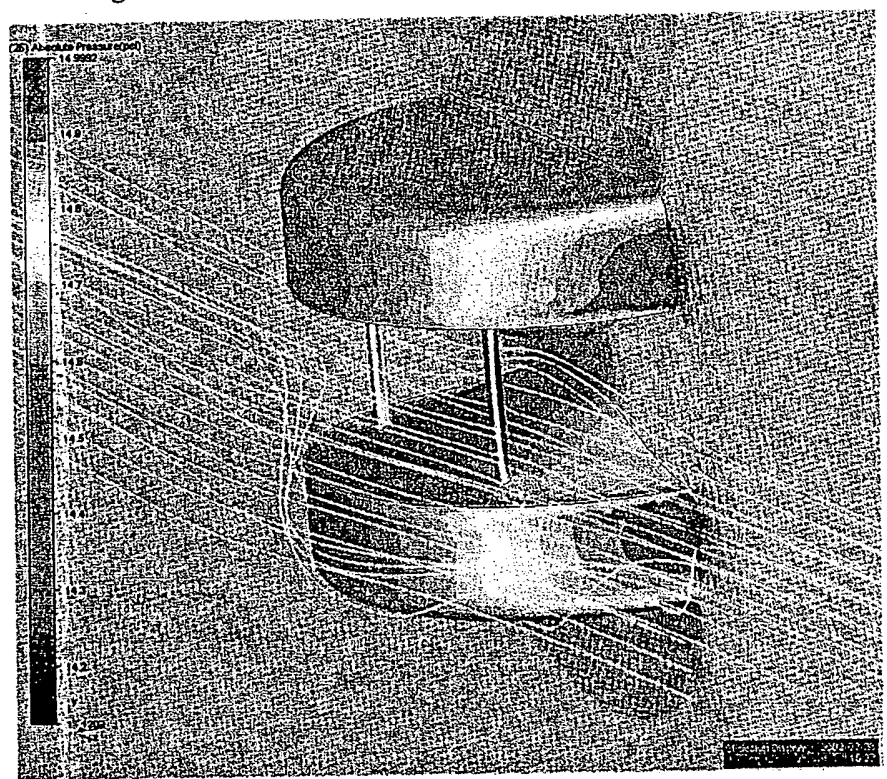

Figure 3. - Wind Pressure Profile from CFD Simulation

A conceptual approach has been devised to create a surface wind profile (wind velocity and direction) sensor by combining pressure measurements, smart software algorithms and incorporating the knowledge developed using Computational Fluid Dynamics (CFD) simulation.

The result is a design which incorporates many beneficial qualities that make the $3 \mathrm{D}$ venturi an attractive augmentation to the current equipment. The $3 \mathrm{D}$ venturi has a relatively small profile and no moving parts. It has a fast response, wide dynamic range, and short recovery time and incorporates both speed and directionality of wind, an inherent advantage over cup-and-vane anemometers. Furthermore, the design will be capable of autonomously acquiring and storing data during a storm 
for further analysis. The following milestones have been accomplished:

- First prototype built and tested in a low-speed wind tunnel $127 \mathrm{mph}$.

- Modeling, analysis and simulation of design at high wind velocities $(\sim 300 \mathrm{mph})$ using Computational Fluid Dynamics (CFD) software has been completed.

The following objectives are lined up for the project:

- Validate design in high-speed wind tunnel at extreme wind velocities to $300 \mathrm{mph}$.

- Conceptualize and Integrate methodology for wind direction determination.

- Optimize port locations to achieve best sensitivity and dynamic response.

- Optimize design to provide remote, standalone system capable of autonomously acquiring, recorling, and storing storm information.

- Ruggedize the design for field deployment.

- Field deploy and test the system.

\section{NON-INVASIVE SENSORS, EMBEDDED KNOWL IDGE IN SENSORS}

A main effort, which is being presently pursued, s the development of a Valve Health Monitor (VHM) $s$ :nsor, also referred as Smart Current Signature Sensor (SC; ;S).

The development of the Valve Health Monitor ( / / HM) sensor is headed by the above-mentioned authors $\mathrm{i} .1$ conjunction with Mr. Angel Lucena of NASA-KSC al d Mr. Bradley Burns of DYNACS Incorporated.

Several hundred solenoid-operated valves are used : round KSC to aid in the storage and distribution of high as d low pressure gases, fuel and oxidizer fluids, and other commodities for ground and flight systems required at li unch. Since some of these systems are critical in nature, $t$ ere is a requirement to provide highly reliable non-in'asive monitoring of these systems. Two main require nents drove the development of this project:

- Be non-invasive. The sensor will monitor the d ssired parameter without interfering with ground or flight systems.

- Monitor the parameter and predict the perfori jance and health of the device. Ultimately, to predict I failure before it happens.

Commonly, the parameters monitored in a solenoid ralve are voltage and current. Since the sensor has to be noninvasive, the magnetic field generated by the current $i$ i the solenoid power leads was selected as the parameter is be monitored. There was no access to monitor the solc noid voltage.
The design presented here monitors the solenoid valve's electrical current for health status and prediction. Both the steady state and the "TURN ON" (Figure 4) and "TURN OFF" (Figure 5) transitions of the current signal are monitored.

The solenoid's current presents unique characteristics (signatures), especially during these transitions. They have very repeatable characteristic peaks and valleys. They happen at a very defined times and have very defined current magnitude and shape. As electrical and/or mechanical degradation occurs in the solenoid valve, the signature changes both in time and magnitude, thus becoming a clear indicator of potential problems.

The sensor is composed of a signal acquisition assembly (Figure 6) and a signal conditioner and controller assem-

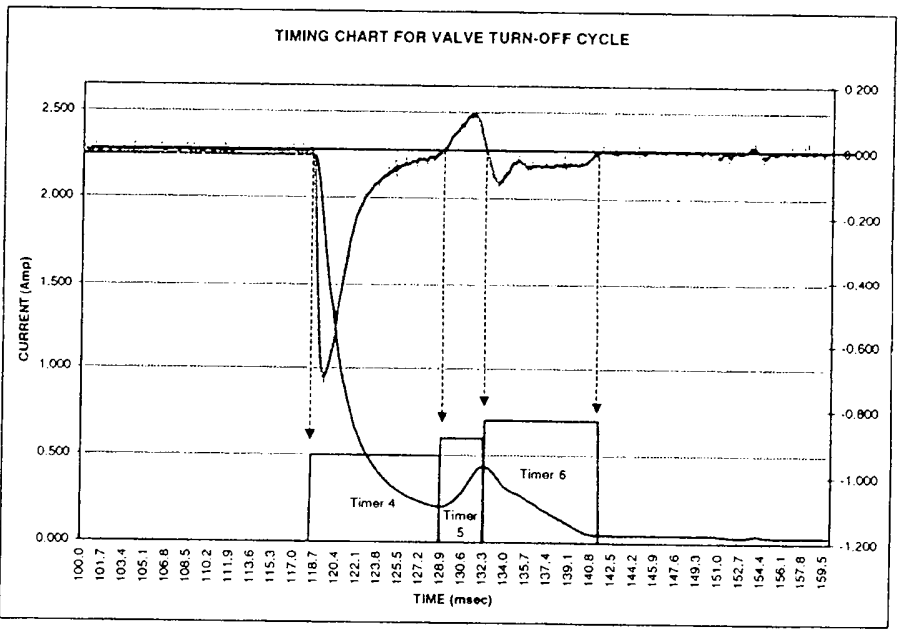

Figure 4. - Solenoid Current Signature (TURN-ON)

bly (Figure 7). The signal acquisition assembly contains the magnetic and temperature sensors. The magnetic sensor selected is a linear Hall effect sensor. To maximize the magnetic field (flux) in the Hall effect sensing area, a flux concentrator is used. To prevent external magnetic fields interference, a magnetic shielding cage is built around the sensor.

One of the major obstacles encountered when using Hall effect sensors is their sensitivity to environment's temperature. As temperature changes, drifts in the offset voltage and sensitivity of the sensor can be observed. Furthermore, the measured drifts, although predictable for each sensor, change from sensor to sensor. Our design approaches this problem in a unique way. The design presented here is capable to compensate real-time for these parameter variations. A temperature sensor is built into the signal acquisition assembly and used to temperature compensate the offset and sensitivity (gain) drifts of the magnetic sensor.

The signal conditioner/controller assembly will provide the following functions: 
- Amplification of the low-level signal from the :signal acquisition assembly into a usable level.

- Continuous real-time temperature compensation of sensor offset and sensitivity (gain) drift.

- Real-time in-circuit calibration of the Current Signature sensor.

- Real-time current signature analysis and trend analysis.

- Perform alert/annunciation when functional de gradation or a failure of the valve (and/or solenoir1) occurs.

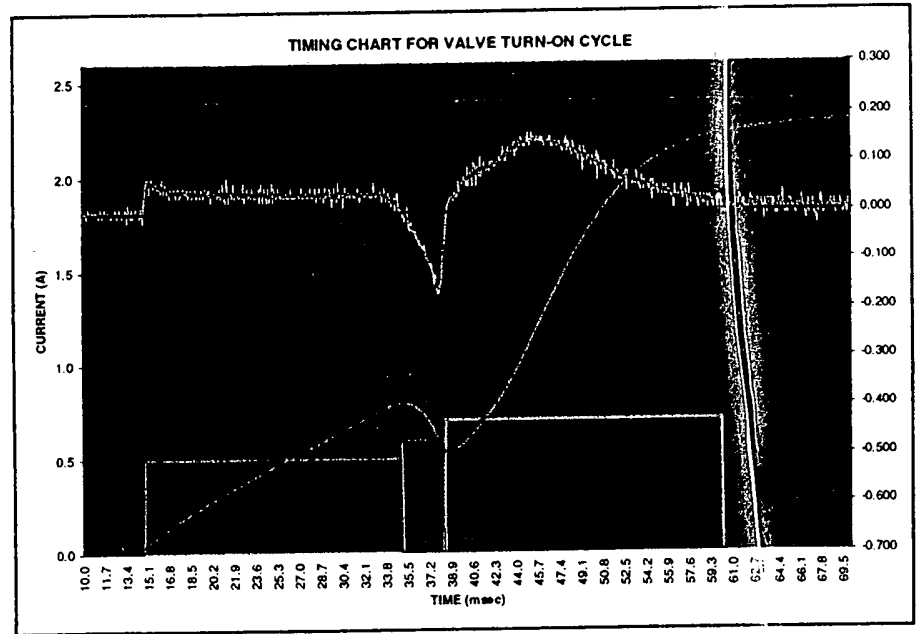

Figure 5. - Solenoid Current Signature (TURN-C)FF)

Valve health analysis and failure prediction will be performed using software algorithms residing in the microprocessor controller module. Information on the chirracteristics (signatures) of the current signal will be extracted from the analog signal and compared against stored parameters of a typical solenoid behavior for that family of valves. The comparisons performed will be gradecl as nominal, borderline or failure. An account of these results will be stored as well as forwarded to the user for further action.

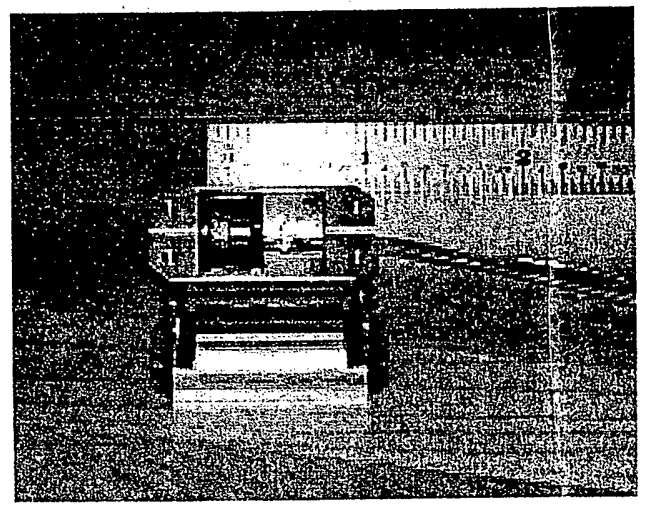

Figure 6. - Signal Acquisition Head (prototype)
A simple algorithm has been devised to detect specific characteristics in the current signal. A derivative approach of the time-domain signal is calculated in realtime. Peaks and valleys of the current signal are detected and time-tagged by looking for the signal transitions from positive to negative and vice versa (zero-crossing transitions). Signal slope and steady state values are also monitored to complete the characterization of the current signature. The following milestones have been accomplished:

- Continuous real-time compensation of offset and sensitivity (gain) drifts over a wide temperature variation.

- Preliminary smart software algorithm has been coded and tested.

The following objectives are planned for the project:

- Perform real-time current signature analysis and trend analysis.

- Develop capability for real-time calibration of the Current Signature sensor.

- Develop valve signature database and capability to inform user if functional degradation or failure of the valve has occurred.

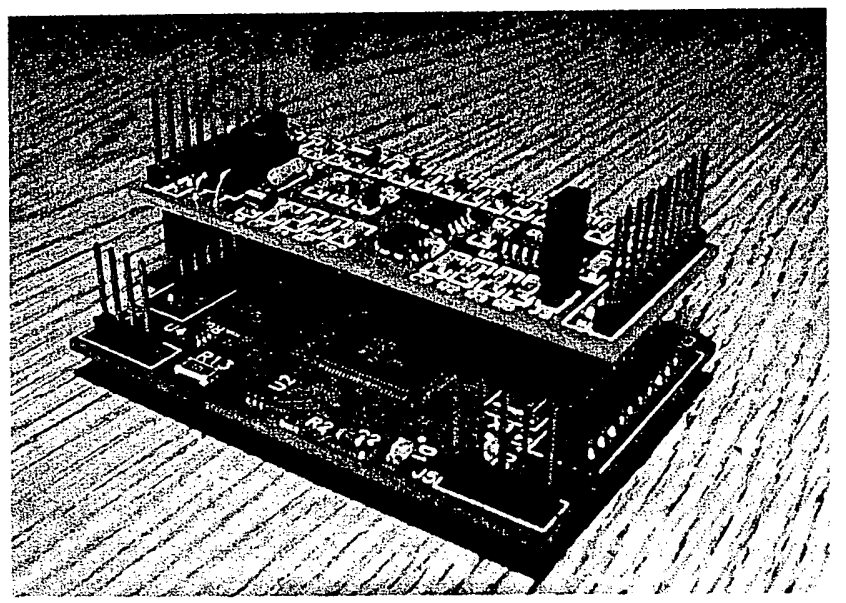

Figure 7. - Signal Conditioner \& Control Module (prototype)

\section{WIRELESS SENSOR NETWORK}

A main effort is being presently pursued related to this discipline: the development of wireless sensor network architecture to support KSC needs. So far, two specific applications have spun out of this effort: the Wireless Vacuum Jacketed (VJ) sensor network (to monitor vacuum of cryogenic lines) and the External Tank (ET) wireless centering and alignment system.

The development of the Wireless Sensor Network is headed by the above-mentioned authors in conjunction with Mr. Angel Lucena of NASA-KSC and Dr. Pedro Medelius, Dr. Carlos Mata, Mr. Norman Blalock and Mr. Bradley Burns of DYNACS Incorporated. Wireless sensor networks are attractive to aerospace applications because 
they do not have the additional weight, size, required support structure (cable trays and bulkheads), and constant maintenance of wired systems. They are also easily reconfigurable. Wireless networks have been available in the market for a while and their benefits well demonstrated. Innovative approaches like embedding wireless capabilities in sensors are rapidly emerging.

The Transducers group at KSC started development wireless technology about two years ago. The main goals in this project were (a) to integrate wireless communication in the sensors and transducers, and (b) to embed process knowledge in these sensors and to share that knowledge through out the network.

The Space Shuttle Program requirements prompted additional implementation of innovative ideas. First, there is a requirement to maintain EMC, RFI interferences to a minimum at the Pad. To do that, RF output power was kept to a minimum $(\leq 10 \mathrm{~mW})$. Since data availability was also a requirement, an innovative software (Lost Station Algorithm) approach was created to allow alternate communication routes for the sensors (embedded intelligence in the sensors) in case of interferences or loss of communication. Additionally, capability to use remote stations as data relay stations was necessary due to the long distances to be covered. Finally, an innovative power management algorithm was created to support operations at the Launch Pad for two years before replacing batteries.

The basic Wireless Sensor Network architecture has a number of remote stations (integrated in sensors), responsible for collecting, analyzing, and transmitting sensor and process information. It also has one or several central stations, responsible for receiving the sensors' information and distributing this information to the users.

Each remote unit of the Wireless Sensors Network consists of a transducer specific module, a processor, an RF transceiver, an antenna, a power management module, and

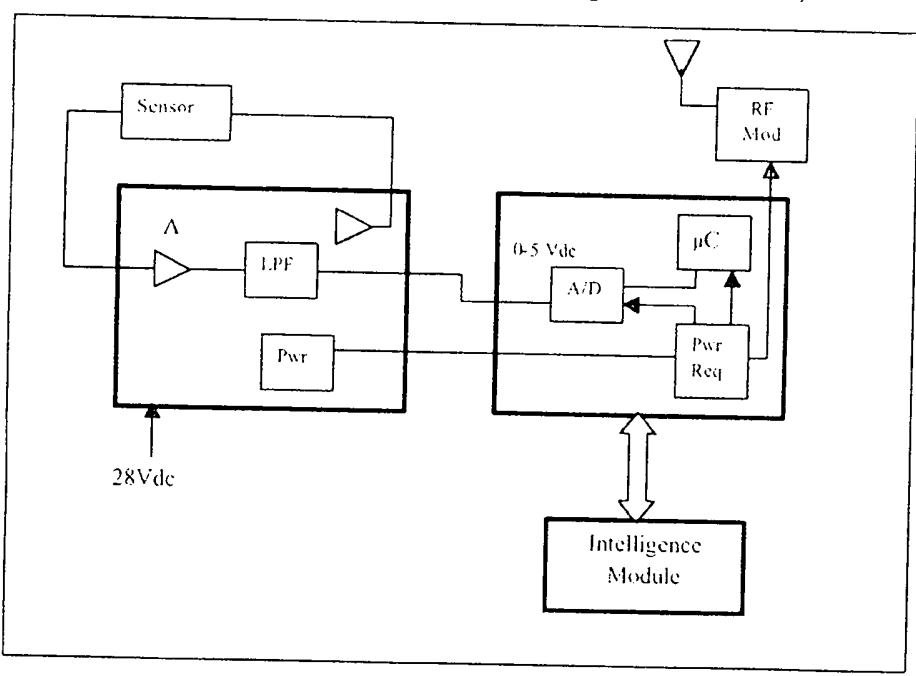

Figure 8. - Remote Station Architecture environmental protective enclosure (Figure 8). The wireless remote units have been designed modular, so they are able adapt to future installation requirements, as well as being sapable of working as a replacement unit for existing har d wire devices. They have been designed in such a manne: that they can be installed in the existing system utilizirg available 28 VDC supply power or have selfcontained battery power. All measurement information is transinitted back to a central station and sent to the central data gathering equipment or processed through a host comp iter. The central station polls the remote processor in orler to acquire information from each of the remote statio is on periodic cycle. The received data is processed and displayed on the central station computer. Software conta ned in the central station is responsible for information $\mathrm{g}$ athering, analysis, and distribution to users. Additional $y$, it contains control software to manage the network iraffic as well as potential communication problems. The "Master Configuration Window" controls all remote static $n$ selection, configuration, data summary, and status infor nation. Once a Remote Station ID is selected and activated, the particular ID number appears in the top righ! "ID Data Units" window. Additionally, a "Remote Stat on Window" display opens to allow configuration of data scaling / engineering unit conversion and sample. Thi: display allows for either raw data or scaled data to be dis\} layed; the selection of the "Scaled Data" button allow $s$ for gain and offset values to be applied to measured rau data.

Th: "Summary" window allows for a summarized assess$\mathrm{m} n \mathrm{nt}$ of the number of active remote stations, etc (seven rc note stations active). The "Status" window provides a St : nmarized assessment of the condition of the system and re note station communication (i.e., four lost stations exist, tv o stations not responding, etc).

A list of active remote stations is contained in the "ID D ata Units" window, along with actual data received and tl e applicable engineering units. A remote station is ren oved from the list by selecting the appropriate ID num$\mathrm{t}$ ar and pressing the "Close" button, which subsequently c loses the "Rt:mote Station Window" display for that ID.

\section{SUMMARY}

The Transdu ers and Data Acquisition Group at KSC continues eff c rts to study, research, develop and prototype inexpensive sı nart sensors to accomplish space transportation system pirformance improvements at reduced operational costs. Technological challenges, in the areas of multi-disciplir e sensors; sensor-embedded selfcalibration, se If-health and self-diagnosis capabilities; and advanced dati acquisition systems with failure prediction algorithms ard failure correction (self-healing) capabilities, continue to be pursued with significant success. 